\title{
Existencia de soluciones estacionarias para un fluido compresible isotérmico
}

\author{
Yony Raúl Santaria Leuyacc ${ }^{1}$ y José Kenyn Rodriguez Briceño ${ }^{2}$
}

Resumen: En este trabajo nos interesa estudiar la existencia de soluciones estacionarias para una ecuación de Navier-Stokes-3D en un fluido compresible e isotérmico. Para probar la existencia de soluciones usaremos un método de aproximaciones sucesivas, siguiendo los resultados mostrados por M. Padula.

Palabras clave: Navier-Stokes; ecuación estacionaria; gases; método de aproximaciones sucesivas.

\section{Existence of stationary solutions for an isothermal compressible flow}

\begin{abstract}
In this work we are interested in studying the existence of stationary solutions for a Navier-Stokes-3D equation in a compressible and isothermal flow. In order to prove the existence of solutions we use a method of successive approximations, following the results shown by M. Padula.
\end{abstract}

Keywords: Navier-Stokes; stationary equation; gases; method of successive approximations.

Recibido: 12/11/2019. Aceptado: 16/07/2020. Publicado online: 18/08/2020.

${ }^{2}$ UNMSM, Facultad de Ciencias Matemáticas, e-mail: jose.rodriguez4@unmsm.edu.pe 


\section{Introducción}

Uno de los problemas más interesantes y de mayor dificultad en el estudio teórico-matemático de las ecuaciones en derivadas parciales que surgen de la mecánica de fluidos es sobre las ecuaciones que rigen el movimiento de un fluido compresible, esto es, cuando un fluido presenta una variación significativa en su densidad, como sucede por ejemplo en los gases. Una de las principales dificultades que se presenta en el flujo de este tipo de fluidos, es su naturaleza genuinamente no lineal regido por ecuaciones de tipo mixtas hiperbólicas-parabólicas.

En la literatura sobre las ecuaciones de Navier-Stokes, existen varios resultados para el caso de un fluido incompresible, es decir, para un fluido donde el volumen ocupado por una cantidad de partículas no varía con el tiempo. Esta condición se traduce en la siguiente propiedad para el campo de velocidades

$$
\nabla \cdot u=0
$$

dicha restricción supone una ventaja importante: la presión (comúnmente denotada por $p$ ) desaparece en la formulación variacional del problema, con lo que deja de ser una incógnita. Así, una vez resuelto el sistema (y, por tanto, conocido el campo de velocidades $u$ ), la presión es recuperada como consecuencia del Lema de De Rham (véase $[10,16,11]$ entre otros).

Sin embargo, no sucede lo mismo para el caso compresible. No sólo permanece $p$ en la formulación variacional, sino que la ecuación de momentos y la ley de conservación de la masa estarán acopladas.

Debido a la complejidad de este tipo de EDP's, se estudiará el comportamiento de un fluido compresible isotérmico, como puede ser un gas, o una masa de aire. Para los gases se considera ecuaciones relacionadas a la termodinámica lo que permiten considerar esta característica física en nuestro problema. Además, se considerará una condición de viscosidad en un ambiente tridimensional, procurando probar la existencia de soluciones para el caso estacionario, es decir, las fuerzas físicas involucradas no dependen del tiempo.

Este hecho permite aproximar la dinámica del comportamiento de dichos sistemas para el caso parabólico e hiperbólico. Una aplicación visible del presente estudio, es el comportamiento de una masa de aire en un ambiente cerrado, así se podrá conseguir aproximaciones del campo de velocidades de dicha masa, como de su densidad, a partir de las fuerzas externas participantes.

Las ecuaciones que rigen el movimiento estacionario de un fluido compresible, isotermo y viscoso en un dominio acotado fijado $\Omega \subset \mathbb{R}^{3}$ se pueden escribir como sigue:

$$
\left\{\begin{array}{r}
-\nabla \cdot\left(\mu\left(\nabla v+\nabla v^{t}\right)+\lambda(\nabla \cdot v) I d\right)+\nabla p+\rho v \cdot \nabla v=\rho f \text { en } \Omega, \\
\nabla \cdot(\rho v)=0 \text { en } \Omega .
\end{array}\right.
$$

Dicho sistema es obtenido a partir del estudio de la segunda ley de Newton y el estudio de conservación de masa donde $\rho$ es la densidad, $v$ la velocidad, $f$ son las fuerzas externas, $p$ es la presión, cuya expresión está dada por la ecuación de estado para un fluido isotermo:

$$
p=K \rho,
$$

con $K>0$ constante y $\mu, \lambda$ siendo los llamados coeficientes de Lamé (que serán considerados constantes).

Es importante notar que al estudiar este fenómeno físico, se requiere ciertas condiciones adicionales respecto al flujo cinético y másico (cf. [17, 3, 16]), así, se considerará

$$
\left.v\right|_{\partial \Omega}=0, \quad \int_{\Omega} \rho(x) d x=\bar{\rho} \cdot|\Omega|
$$


donde $\bar{\rho}>0$ es una constante fijada, que representará la positividad de la masa total y la condición de adherencia sobre la frontera respectivamente (estamos suponiendo por ejemplo una frontera sólida, sin entrada ni salida de fluido).

El objetivo principal de este trabajo será probar la existencia de soluciones estacionarias para el problema (14), esto es un sistema del tipo

$$
\left\{\begin{array}{r}
-\mu \Delta v-\left(\zeta+\frac{\mu}{3}\right) \nabla(\nabla \cdot v)+K \nabla \rho+(\rho v \cdot \nabla) v=\rho f \text { en } \Omega \\
\nabla \cdot(\rho v)=0 \text { en } \Omega .
\end{array}\right.
$$

Bajo las condiciones (2). Siendo $\zeta=\lambda+\frac{2}{3} \mu$, el módulo de compresibilidad, donde consideraciones termodinámicas conducen a dicha restricción. Para probar la existencia de soluciones estacionarias se seguirán los diferentes métodos usados por M. Padula [13].

\section{Aspectos matemáticos sobre la dinámica de fluidos}

En esta sección se detallará las diferentes herramientas matemáticas necesarias para estudiar un problema de existencia de soluciones para fluidos compresibles. Para esto se rescataran diferentes resultados clásicos dentro de esta rama de estudio, para mayor detalle el lector interesado puede consultar $[16,10,2,8,1,9,7,11,19,18]$ entre otros.

El marco de resolución de las ecuaciones serán los espacios de Lebesgue $L^{p}(\Omega)$ y Sobolev $W^{m, p}(\Omega)$ ( $m$ entero, $\left.1 \leq p \leq+\infty\right)$ usuales con las normas estándar $|\cdot|_{p},|\cdot|_{m, p}$ respectivamente.

Definimos los espacios de Sobolev con media nula, como:

$$
W_{M}^{m, p}(\Omega)=\left\{\psi: \psi \in W^{m, p}(\Omega) \text { tal que } \int_{\Omega} \psi d x=0\right\}
$$

dotado de la misma norma que $W^{m, p}(\Omega)$. A continuación enunciaremos una desigualdad importante para estos espacios el cual puede ser encontrado en [11].

Lema 2.1 (Desigualdad de Poincaré para funciones con media nula) Sea $\partial \Omega \in \mathcal{C}^{0,1}(\Omega)$ y $\psi \in W_{M}^{1, p}(\Omega)$. Entonces:

$$
|\psi|_{1, p} \leq C_{1}\left(\sum_{n=1}^{3}\left|\frac{\partial \psi}{\partial x_{i}}\right|_{p}^{p}\right)^{1 / p}
$$

donde $C_{1}$ es una constante positiva que solo depende de $\Omega$ y p.

Consideramos el problema de Stokes no homogéneo en $\Omega$. El cual jugará un rol fundamental en la prueba de existencia de soluciones para el sistema (1) a partir de la descomposición en un sistema de Stokes equivalente.

$$
\left\{\begin{array}{l}
-\Delta \varphi+\nabla \varphi=F, \nabla \cdot \varphi=g \\
\left.v\right|_{\partial \Omega}=0
\end{array}\right.
$$

Lema 2.2 (Cattabriga-Solonnikov) Sea $\partial \Omega$ de clase $\mathcal{C}^{m}, F \in\left(W^{m-2, q}(\Omega)\right)^{3}$ y $g \in W^{m-1, p}(\Omega)$ $(m \geq 1,1<q<\infty)$ tal que verifica la condición de compatibilidad:

$$
\int_{\Omega} g(x) d x=0
$$


Entonces existe una y solo una solución $(\varphi, \pi) \in\left(W^{m, q}(\Omega)\right)^{3} \times W^{m-1, q}(\Omega)$ de (5) ( $\pi$ es única salvo constantes aditivas). Además, se tiene la siguiente estimación de dependencia continua de la solución $(\varphi, \pi)$ respecto de los datos $(F, g)$ :

$$
|\varphi|_{m, q}+|\nabla \pi|_{m-2, q} \leq C_{2}\left(|F|_{m-2, q}+|g|_{m-1, q}\right)
$$

donde $C_{2}$ es una constante positiva que solo depende de $m, q$ y .

El lema anterior será de vital importancia en la prueba del resultado principal en el presente trabajo, al permitir garantizar la existencia de soluciones para el problema de Stokes asociado.

Por otro lado, será necesario un resultado de existencia para el control del flujo másico. A continuación se describe dicho resultado, por comodidad del lector se indicará su prueba en detalle.

Lema 2.3 Sea L el operador diferencial lineal de primer orden

$$
L \psi=a \psi+\nabla \cdot(b \psi),
$$

donde $a>0$ es una constante y $b \in\left(W^{2,4}(\Omega)\right)^{3} \cap\left(H_{0}^{1}(\Omega)\right)^{3}$. Consideramos que L actúa sobre funciones escalares $\psi$ definidas en $\Omega$, y consideramos

$$
D(L)=\left\{\psi \in W^{1,4}(\Omega): L \psi \in W^{1,4}(\Omega) \text { y } \int_{\Omega} \psi(x) d x=0\right\} .
$$

Si

$$
a-7 C_{4} C_{1}|\nabla(\nabla \cdot b)|_{4}-4 C_{4}|b|_{2,4}>0
$$

para $C_{4}$ la constante tal que $|\psi|_{\infty} \leq C_{4}|\psi|_{1,4}$ (es decir, la constante de la inyección continua de Sobolev de $W^{1,4}(\Omega)$ en $\mathcal{C}^{0}(\bar{\Omega})$ ). Entonces, para cada $G \in W^{1,4}(\Omega)$ con $\int_{\Omega} G d x=0$, existe una única solución $\psi \in D(L)$ de

$$
L \psi=G
$$

\section{Demostración.}

Debido a la linealidad del operador diferencial, la existencia y unicidad en el espacio $H^{1}(\Omega)$ con medida nula se seguirá de forma estándar si conseguimos estimaciones a priori en dicho espacio.

Aplicando un razonamiento de tipo Galerkin, eligiendo como base especial en $H^{1}(\Omega)$ una base $\left\{z_{1}, z_{2}, \ldots, z_{m}, \ldots\right\}$ de autovectores del problema:

$$
\left\{\begin{aligned}
-\Delta z+z=\lambda z & \text { en } \Omega \\
\frac{\partial z}{\partial n}=0 & \text { sobre } \partial \Omega
\end{aligned}\right.
$$

cuya formulación variacional es:

$$
z \in H^{1}(\Omega) \text { tal que }((z, w))=\lambda(z, w), \forall w \in H^{1}(\Omega),
$$

donde $((\cdot, \cdot))$ es el producto escalar en $H^{1}(\Omega)$ y $(\cdot, \cdot)$ es el producto escalar en $L^{2}(\Omega)$.

Para demostrar la existencia de dicha base, consideramos el operador

$$
T: f \in L^{2}(\Omega) \rightarrow z \in H^{1}(\Omega)
$$


donde $z(f)$ es la solución del problema variacional:

$$
((z, w))_{H^{1}}=(f, w)_{L^{2}}, \forall w \in H^{1}(\Omega) .
$$

Como $H^{1}(\Omega) \stackrel{c}{\hookrightarrow} L^{2}(\Omega)$ (inmersión compacta), entonces

$$
T: L^{2}(\Omega) \rightarrow L^{2}(\Omega) \text { es un operador compacto. }
$$

Además,

$$
(f, T g)_{L^{2}}=(z, T g)_{H^{1}}=(T g, z)_{H^{1}}=(g, z)_{H^{1}}=(z, g)_{L^{2}}=(T f, g)_{L^{2}}, \quad \forall f, g \in L^{2}(\Omega) .
$$

Luego, $T$ es un operador autoadjunto.

Por otro lado, siendo $L^{2}(\Omega)$ un Hilbert separable, en virtud del Teorema de Hilbert-Schmidt (véase [14, Teorema 8.94]), podemos garantizar la existencia de una base ortonormal en $L^{2}(\Omega)$, $\left\{z_{1}, z_{2}, \ldots, z_{m}, \ldots\right\}$ formada por autovectores de $T$. Asimismo, dicha base también es una base ortogonal de $H^{1}(\Omega)$.

Dada $G \in W^{1,4}(\Omega)$, considere el problema:

$$
\text { hallar } \psi \in H^{1}(\Omega) \text { tal que } a \psi+\nabla \cdot(b \psi)=G,
$$

es decir,

$$
\psi \in H^{1}(\Omega) \text { tal que } a(\psi, \varphi)-\int_{\Omega} \psi b \cdot \nabla \varphi=(G, \varphi), \forall \varphi \in H^{1}(\Omega) .
$$

Donde $b=0$ en la frontera de $\Omega$ por definición.

Planteando el problema discretizado:

$$
\psi_{m} \in\left\langle z_{1}, \cdots, z_{m}\right\rangle, a\left(\psi_{m}, \varphi\right)-\int_{\Omega} \psi_{m} b \cdot \nabla \varphi=(G, \varphi), \varphi \in\left\langle z_{1}, \cdots, z_{m}\right\rangle,
$$

donde

$$
\psi_{m}=\sum_{j=1}^{m} \alpha_{j} z_{j} \quad \text { donde } \quad \varphi=z_{i}, \alpha_{i} \in \mathbb{R}, i=1, \ldots, m,
$$

se deduce que (9) es equivalente al sistema lineal:

$$
\sum_{j=1}^{m}\left\{\alpha_{j} a\left(z_{j}, z_{i}\right)-\alpha_{j}\left(\int_{\Omega} z_{j} b \cdot \nabla z_{i}\right)\right\}=\left(G, z_{i}\right), \quad i=1, \ldots, m .
$$

La matriz del sistema es:

$$
A=\left(a\left(z_{j}, z_{i}\right)-\int_{\Omega} z_{j} b \cdot \nabla z_{i}\right)_{i, j=1}^{m} \in \mathbb{R}^{m \times m} .
$$

Véase que $A$ es definida positiva. En efecto, dado $\alpha \in \mathbb{R}^{m}$, si se considera $\psi_{m}$, como en (10),

$$
(A \alpha, \alpha)=a\left|\psi_{m}\right|_{2}^{2}+\frac{1}{2} \int_{\Omega}(\nabla \cdot b) \psi_{m}^{2}
$$

Como

$$
\int_{\Omega}(\nabla \cdot b) \psi_{m}^{2} \leq|\nabla \cdot b|_{\infty}\left|\psi_{m}\right|_{2}^{2} \leq C_{4}|\nabla \cdot b|_{1,4}\left|\psi_{m}\right|_{2}^{2} \leq C_{4} C_{1}|\nabla(\nabla \cdot b)|_{4}\left|\psi_{m}\right|_{2}^{2}
$$


se obtiene

$$
(A \alpha, \alpha) \geq\left(a-\frac{1}{2} C_{4} c_{1}|\nabla(\nabla \cdot b)|_{4}\right)\left|\psi_{m}\right|_{2}^{2}
$$

De la condición (6), en particular, $a-C_{4} C_{1}|\nabla(\nabla \cdot b)|_{4}>0$. Por tanto, el problema discreto (9) tiene una única solución $\psi_{m}$. Además, tomando en $(9)$, como función prueba $\varphi=\psi_{m}$ :

$$
a\left|\psi_{m}\right|_{2}^{2}+\frac{1}{2} \int_{\Omega}(\nabla \cdot b) \psi_{m}^{2}=\left(G, \psi_{m}\right) \leq \frac{a}{2}\left|\psi_{m}\right|_{2}^{2}+\frac{1}{2 a}|G|_{2}^{2} .
$$

Análogamente, repitiendo el mismo proceso se tiene la siguiente estimativa de $\psi_{m}$ y utilizando la desigualdad de Young, se obtiene

$$
\left|\psi_{m}\right|_{2}^{2} \leq \frac{|G|_{2}^{2}}{a\left(a-C_{4} C_{1}|\nabla(\nabla \cdot b)|_{4}\right)}
$$

En consecuencia, debido a la inmersión compacta entre los espacios se puede extraer una subsucesión $\left(\psi_{m^{\prime}}\right)_{m^{\prime}}$ tal que $\psi_{m^{\prime}} \rightarrow \psi$ en $L^{2}(\Omega)$ débil. Además, pasando al límite en (9), se tiene que $\psi$ es solución de (8) y $\int_{\Omega} \psi d x=0$; para ello, basta tomar $\psi \equiv 1$ en (8) y considerar $\int_{\Omega} G=0$.

De este modo se obtiene una solución de $(7)$ en $H^{1}(\Omega)$. La unicidad es consecuencia del operador diferencial, lo que lo permite comparar fácilmente la linealidad de los operadores.

Para terminar la demostración del lema, observe que $\psi \in W^{1,4}(\Omega)\left(\operatorname{como} H^{1}(\Omega) \hookrightarrow L^{4}(\Omega)\right.$, basta ver que $\left.\nabla \psi \in\left(L^{4}(\Omega)\right)^{3}\right)$. Luego, de la ecuación $L \psi=G$, se obtiene

$$
\begin{aligned}
&|\nabla G|_{4}^{4}=a^{4}|\nabla \psi|_{4}^{4}+|\nabla(\nabla \cdot(b \psi))|_{4}^{4}+2 \int_{\Omega} a^{2}|\nabla \psi|^{2}|\nabla(\nabla \cdot(b \psi))|^{2} d x \\
&+4 \int_{\Omega} a^{2}|\nabla \psi \cdot \nabla(\nabla \cdot(b \psi))|^{2} d x+4 \int_{\Omega} a^{3}|\nabla \psi|^{2} \nabla \psi \cdot \nabla(\nabla \cdot(b \psi)) d x \\
&+4 \int_{\Omega} a \nabla \psi \cdot \nabla(\nabla \cdot(b \psi))|\nabla(\nabla \cdot(b \psi))|^{2} d x
\end{aligned}
$$

Empleando las desigualdades de Schwartz, Hölder y Young se deducen

$$
\begin{gathered}
\int_{\Omega}|\nabla \psi|^{2}|\nabla(\nabla \cdot(b \psi))|^{2} \geq \int_{\Omega}[\nabla \psi \cdot \nabla(\nabla \cdot(b \psi))]^{2} d x \\
\int_{\Omega} a \nabla \psi \cdot(\nabla \cdot(b \psi))|\nabla(\nabla \cdot(b \psi))|^{2} d x \geq-\frac{1}{6}|\nabla(\nabla \cdot(b \psi))|_{4}^{4}-\frac{3}{2} a^{2}|\nabla \psi \cdot \nabla(\nabla \cdot(b \psi))|_{2}^{2}
\end{gathered}
$$

y

$$
\begin{aligned}
\int_{\Omega}|\nabla \psi|^{2} & \nabla \psi \cdot \nabla(\nabla \cdot(b \psi)) d x \\
& =\int_{\Omega}|\nabla \psi|^{2} \nabla \psi \cdot[b \cdot \nabla(\nabla \psi)+\psi \nabla(\nabla \cdot b)+(\nabla b)(\nabla \psi)+(\nabla \cdot b)(\nabla \psi)] d x \\
& \geq-\frac{1}{4} \int_{\Omega}|\nabla \psi|^{4}(\nabla \cdot \psi) d x-C_{4}|\nabla(\nabla \cdot b)|_{4}|\nabla \psi|_{4}^{4}-|\nabla b|_{\infty}|\nabla \psi|_{4}^{4}+\int_{\Omega}(\nabla \cdot b)|\nabla \psi|^{4} d x \\
& \geq-\left(\frac{3}{4}|\nabla \cdot b|_{\infty}+|\nabla b|_{\infty}+C_{4} C_{1}|\nabla(\nabla \cdot b)|_{4}\right)|\nabla \psi|_{4}^{4}
\end{aligned}
$$


Luego, de (12), (13) y (14), se concluye que

$$
\begin{aligned}
|\nabla G|_{4}^{4} & \geq a^{3}\left\{a-4\left(\frac{3}{4} C_{4} C_{1}|\nabla(\nabla \cdot b)|_{4}+C_{4}|b|_{2,4}+C_{4} C_{1}|\nabla(\nabla \cdot b)|_{4}\right)\right\}|\nabla \psi|_{4}^{4} \\
& \geq a^{3}\left\{a-7 C_{1} C_{4}|\nabla(\nabla \cdot b)|_{4}-4 C_{4}|b|_{2,4}\right\}|\nabla \psi|_{4}^{4} .
\end{aligned}
$$

Finalmente, usando (6), se tiene que $\nabla \psi \in\left(L^{4}(\Omega)\right)^{3}$, lo que prueba el resultado.

\section{Soluciones estacionarias para un fluido compresible isotérmi- co}

Esta sección esta destinada a mostrar el resultado principal del presente trabajo, es decir, mostrar la existencia de soluciones para el sistema (1) bajo las condiciones (2), en un ambiente tridimensional.

Consideraremos las constantes termodinámicas con las siguientes restricciones, debido a que el gas es isotérmico

$$
\mu>0, \quad 3 \lambda+2 \mu \geq 0 .
$$

Lo que permite reescribir (1) a partir del cambio de variable

$$
\zeta=\frac{3 \lambda+2 \mu}{3}
$$

como

$$
\left\{\begin{aligned}
-\mu \Delta v-\left(\zeta+\frac{\mu}{3}\right) \nabla(\nabla \cdot v)+K \nabla \rho+(\rho v \cdot \nabla) v=\rho f & \text { en } \Omega \\
\nabla \cdot(\rho v)=0 & \text { en } \Omega .
\end{aligned}\right.
$$

\subsection{Entorno funcional}

Debido a que se quiere estudiar la existencia de soluciones, se precisa de diversas regularidades respecto a las variables a estudiar. A continuación se expondrá el ambiente funcional donde habitan las soluciones estacionarios para el problema (1) $\equiv(14)$.

Dado $\Omega \subset \mathbb{R}^{3}$ un abierto, acotado con frontera regular y $p \in[1, \infty]$, se define el espacio de Banach vectorial $\left(\left(L^{p}(\Omega)\right)^{3},|\cdot|_{p}\right)$ como

$$
\left(L^{p}(\Omega)\right)^{3}:=\left\{v: \Omega \rightarrow \mathbb{R}^{3} \mid v=\left(v_{1}, v_{2}, v_{3}\right) \wedge v_{i} \in L^{p}(\Omega)\right\},
$$

con norma

$$
|v|_{p}^{p}=\sum_{i=1}^{3}\left|v_{i}\right|_{p}^{p},
$$

donde $\left(L^{p}(\Omega),|\cdot|_{p}\right)$ representa al espacio usual $L^{p}(\Omega)$, tal que

$$
\begin{gathered}
\left|v_{i}\right|_{p}^{p}=\int_{\Omega}\left|v_{i}\right|^{p} d x, \quad p \in[1, \infty), \\
\left|v_{i}\right|_{\infty}=\sup \operatorname{ess}\left|v_{i}(x)\right|,
\end{gathered}
$$

siendo sup ess $(\cdot)$ el operador supremo esencial.

Análogamente, se define el espacio de Sobolev con medida nula vectorial $\left(W^{m, p}(\Omega),|\cdot|_{m, p}\right)$ como

$$
\left(W^{m, p}(\Omega)\right)^{3}:=\left\{v: \Omega \rightarrow \mathbb{R}^{3} \mid v=\left(v_{1}, v_{2}, v_{3}\right) \wedge v_{i} \in W^{m, p}(\Omega)\right\},
$$


con norma

$$
|v|_{m, p}^{p}=\sum_{i=1}^{3}\left|v_{i}\right|_{m, p}^{p},
$$

donde $\left(W^{m, p}(\Omega), \mid \cdot{ }_{m, p}\right)$ representa al espacio de Sobolev con medida nula definido en (3), tal que

$$
\left|v_{i}\right|_{m, p}^{p}=\sum_{\alpha \leq|m|}\left|D^{\alpha} v_{i}\right|_{p}^{p}
$$

con $D^{\alpha}$ representa la notación multi-índice para las derivadas parciales de orden $\alpha$.

En el marco de las notaciones anteriores, definiremos la idea de solución estacionaria para el sistema (14).

Definición 3.1 Dado el sistema (14) bajo las condiciones dadas en (2) con $\Omega \subset \mathbb{R}^{3}$ un conjunto abierto, acotado con frontera $\partial \Omega$ de clase, por lo menos, $\mathcal{C}^{2}$. Sea $\zeta \geq 0, \mu>0$ y $f \in\left(L^{4}(\Omega)\right)^{3}$, entonces se dirá que el par $(v, \rho) \in\left(W^{2,4}(\Omega)\right)^{3} \times W^{1,4}(\Omega)$ es una solución estacionaria para el sistema (14)-(2) si y solo si

$$
\begin{gathered}
-\mu \Delta v-\left(\zeta+\frac{\mu}{3}\right) \nabla(\nabla \cdot v)+K \nabla \rho+(\rho v \cdot \nabla) v=\rho f, \\
\nabla \cdot(\rho v)=0,
\end{gathered}
$$

en $\left(L^{4}(\Omega)\right)^{3}$ y $L^{4}(\Omega)$ respectivamente.

La definición anterior está bien definida, dado que si $v \in\left(W^{2,4}(\Omega)\right)^{3}, \rho \in W^{1,4}(\Omega)$ y $f \in$ $\left(L^{4}(\Omega)\right)^{3}$, entonces se observa que

$$
-\mu \Delta v-\left(\zeta+\frac{\mu}{3}\right) \nabla(\nabla \cdot v)+K \nabla \rho+(\rho v \cdot \nabla) v \in\left(L^{4}(\Omega)\right)^{3} .
$$

Además, como $W^{1,4}(\Omega)$ esta inmerso continuamente en $L^{\infty}(\Omega)$, si $f=\left(f_{1}, f_{2}, f_{3}\right)$ entonces para todo $i=1,2,3$ se tiene que

$$
\begin{gathered}
\int_{\Omega} \rho\left|f_{i}\right|^{4} d x \leq|\rho|_{\infty}\left|f_{i}\right|_{4}^{4}<\infty \\
\int_{\Omega} \rho^{2}\left|f_{i}\right|^{4} d x=\int_{\Omega} \rho \rho\left|f_{i}\right|^{4} d x \leq|\rho|_{\infty} \int_{\Omega} \rho\left|f_{i}\right|^{4} d x \leq|\rho|_{\infty}^{2}\left|f_{i}\right|^{4}<\infty
\end{gathered}
$$

lo que análogamente muestra que

$$
\int_{\Omega} \rho^{4}\left|f_{i}\right|^{4} d x \leq|\rho|_{\infty}^{4}\left|f_{i}\right|_{4}^{4}<\infty
$$

es decir, $\rho f \in\left(L^{4}(\Omega)\right)^{3}$.

Por otro lado, razonando de manera similar a lo mostrado anteriormente, si $v=\left(v_{1}, v_{2}, v_{3}\right)$, entonces para todo $i=1,2,3$,

$$
\begin{gathered}
\int_{\Omega}\left|\frac{\partial \rho}{\partial x_{i}} v_{i}\right|^{4} d x \leq\left|v_{i}\right|_{\infty}^{4}\left|\frac{\partial \rho}{\partial x_{i}}\right|_{4}^{4}<\infty \\
\int_{\Omega}\left|\frac{\partial v_{i}}{\partial x_{i}} \rho\right|^{4} d x \leq|\rho|_{\infty}^{4}\left|\frac{\partial v_{i}}{\partial x_{i}}\right|_{4}^{4}<\infty
\end{gathered}
$$

es decir $\nabla \cdot(\rho v) \in L^{4}(\Omega)$. 
Es importante destacar que la Definición 3.1 representa las soluciones fuertes para el sistema (14)-(2). Siendo fundamental la regularidad de la fuerza externa $f$ que gobierna las otras regularidades debido a los productos $\rho f$ y $\nabla \cdot(\rho v)$, por lo que se considera como espacio de fase para $(v, \rho)$ al espacio $\left(W^{2,4}(\Omega)\right)^{3} \times W^{1,4}(\Omega)$.

Otra observación a tener presente para la elección del espacio de fase de las soluciones $\left(W^{2,4}\right)^{3}(\Omega) \times W^{1,4}(\Omega)$ es la dimensión espacial, puesto que $\Omega \subset \mathbb{R}^{3}$, esto permite la inmersión continua de $W^{1,4}(\Omega)$ en $L^{\infty}(\Omega)$ y compacta sobre $L^{p}(\Omega)$ para $p<\infty$. Estos resultados serán fundamentales en la prueba de la existencia de soluciones.

\subsection{Existencia de soluciones y estimativas}

Dado $f \in\left(L^{4}\right)^{3}(\Omega)$, se define las siguientes constantes:

$$
\begin{aligned}
& A_{1}:=\frac{C_{4} C_{2}}{K}|f|_{4}\left\{\frac{49}{\mu} C_{1}+12\left(\frac{\zeta+\mu / 3}{\mu}\right)\right\} \\
& A_{2}:=\frac{7 C_{1} C_{2}}{(\zeta+\mu / 3)}+C_{2} C_{4}|f|_{4}\left\{\frac{4}{K} C_{1}+9 C_{2}\left(\frac{\bar{\rho}}{\mu}\right)^{2}|f|_{4}\left(\frac{1}{2}+\frac{1}{K} C_{1} C_{2} C_{4}|f|_{4}\right)\right\} \\
& A_{3}:=C_{2}|f|_{4}\left\{\frac{4}{K}\left(C_{1} C_{4}+C_{5}\right)\left(\frac{\zeta+\mu / 3}{\mu}\right)+3\left(\frac{\bar{\rho}}{\mu}\right)^{2} C_{2}\left(C_{4}+\left(1+C_{4}\right) \frac{C_{1} C_{2}}{K}|f|_{4}\right)\right\} \\
& A_{4}:=C_{6}\left\{\frac{2}{K}+\frac{C_{2} \mu}{(\zeta+\mu / 3)}\right\} \\
& A_{5}:=C_{2}\left\{\frac{\mu K}{(\zeta+\mu / 3)}+|f|_{4}\left(K_{1}+\frac{9}{2}\left(C_{2} C_{4} \frac{\bar{\rho}}{\mu}\right)^{2}|f|_{4}\right)\right\}
\end{aligned}
$$

donde $K_{1}=|\Omega|^{1 / 4}(|\Omega|$ es la medida de Lebesgue del dominio $\Omega)$ y $C_{5}$ y $C_{6}$ son constantes que se fijarán más adelante.

A continuación enunciamos el resultado principal del presente trabajo.

Teorema 3.2 Dado el sistema (14) bajo las condiciones dadas en (2) con $\Omega \subset \mathbb{R}^{3}$ un conjunto abierto, acotado con frontera $\partial \Omega$ de clase, por lo menos, $\mathcal{C}^{2}$. Sea $\zeta \geq 0, \mu>0$ y $f \in\left(L^{4}(\Omega)\right)^{3}$, tales que se verifican las desigualdades $A_{i}<1, i=1,2, \ldots, 5$, entonces existe al menos una solución estacionaria $(v, \rho) \in\left(W^{2,4}(\Omega)\right)^{3} \times W^{1,4}(\Omega)$ del problema (14)-(2) en el sentido de la Definición 3.1.

Además, dicha solución verifica las siguientes estimativas:

$$
\begin{gathered}
|v|_{2,4} \leq \frac{3 C_{2}}{2} \frac{\bar{\rho}}{\mu}|f|_{4} \\
|\nabla \rho|_{4} \leq 2 C_{2} \frac{\bar{\rho}}{K}|f|_{4} \\
\min _{\Omega} \rho>\frac{\bar{\rho}}{2}
\end{gathered}
$$

Observación 3.3 Las condiciones $A_{i}<1, i=1, \cdots, 5$, establecen restricciones sobre:

(a) $\mu$ y $\zeta$, tales que $\zeta / \mu$ sea suficientemente pequeño.

(b) $K$, tal que $K$ sea suficientemente grande. 
(c) $f$, tal que $|f|_{4}$ sea suficientemente pequeña.

\section{Demostración del Teorema 3.2:}

Realizando el siguiente cambio de variables dependientes:

$$
\left\{\begin{array}{l}
\pi=K \rho-(\zeta+\mu / 3) \nabla \cdot v \\
u=\mu v \\
\sigma=\rho-\bar{\rho},
\end{array}\right.
$$

el sistema (14) y las condiciones adicionales (2) se escriben como

$$
\begin{aligned}
-\nabla u+\nabla \pi & =-\frac{\sigma+\bar{\rho}}{\mu^{2}} u \cdot \nabla u+(\sigma+\bar{\rho}) f \\
\nabla \cdot u & =\frac{\mu}{\zeta+\mu / 3}\{K(\sigma+\bar{\rho})-\pi\} \\
K \sigma+\frac{\zeta+\mu / 3}{\bar{\rho} \mu} \nabla \cdot(\sigma u) & =\pi-\bar{\rho} K, \\
\left.u\right|_{\partial \Omega}=0, \quad \int_{\Omega} \pi d x & =K \bar{\rho}|\Omega| \quad \text { y } \quad \int_{\Omega} \sigma d x=0 .
\end{aligned}
$$

De este modo, el problema se puede dividir en dos subproblemas:

Problema A: Hallar $(u, \pi) \in\left(W^{2,4}(\Omega)\right)^{3} \times W^{1,4}(\Omega)$ tal que:

$$
\left\{\begin{array}{l}
-\Delta u+\nabla \pi=F \\
\nabla \cdot u=\theta \\
\left.u\right|_{\partial \Omega}=0, \int_{\Omega} \pi d x=\bar{\rho} K|\Omega|
\end{array}\right.
$$

donde $F \equiv-\frac{(\sigma+\bar{\rho})}{\mu^{2}} u \cdot \nabla u+(\sigma+\bar{\rho}) f \quad$ y $\quad \theta=\frac{\mu}{(\zeta+\mu / 3)}\{K(\sigma+\bar{\rho})-\pi\}$.

En este problema se satisface $\int_{\Omega} \theta d x=0$.

Problema B: Dado $(u, \pi)$ mediante el Problema A, hallar $\sigma \in W^{1,4}(\Omega)$ tal que:

$$
K \sigma+\nabla \cdot(\sigma w)=G \quad \text { en } \Omega \quad \text { y } \quad \int_{\Omega} \sigma d x=0,
$$

donde $G \equiv \pi-\bar{\rho} K$ y $w=\frac{\zeta+\mu / 3}{\bar{\rho} \mu}$. Observe que $\int_{\Omega} G d x=0$ y $w \in\left(W^{2,4}(\Omega) \cap H_{0}^{1}(\Omega)\right)^{3}$.

A continuación se usará el método de las aproximaciones sucesivas para la búsqueda de solución de ambos problemas. Así, una etapa $n$ del método iterativo se define como: Dados los datos $\left(u_{n-1}, \pi_{n-1}\right) \in\left(W^{2,4}(\Omega)\right)^{3} \times W^{1,4}(\Omega)$ tales que $\int_{\Omega} \pi_{n-1} d x=K \bar{\rho}|\Omega|$ y $\sigma_{n-1} \in W^{1,4}(\Omega)$ tal que $\int_{\Omega} \sigma_{n-1} d x=0$.

Problema $A_{n}$ : Hallar $\left(u_{n}, \pi_{n}\right) \in\left(W^{2,4}(\Omega)\right)^{3} \times W^{1,4}(\Omega)$ solución del problema de Stokes no homogéneo:

$$
\left\{\begin{array}{lc}
-\Delta u_{n}+\nabla \pi_{n}=F_{n-1}, & F_{n-1}=-\frac{\sigma_{n-1}+\bar{\rho}}{\mu^{2}} u_{n-1} \cdot \nabla u_{n-1}+\left(\sigma_{n-1}+\bar{\rho}\right) f \\
\nabla \cdot u_{n}=\theta_{n-1}, & \theta_{n-1}=\frac{\mu}{\zeta+\mu / 3}\left\{K\left(\sigma_{n-1}+\bar{\rho}\right)-\pi_{n-1}\right\} \quad\left(\int_{\Omega} \theta_{n-1}=0\right) \\
\left.u_{n}\right|_{\partial \Omega}=0, & \int_{\Omega} \pi_{n} d x=K \bar{\rho}|\Omega|
\end{array}\right.
$$


Problema $\boldsymbol{B}_{\boldsymbol{n}}$ : Hallar $\sigma_{n} \in W^{1,4}(\Omega)$ tal que:

$$
K \sigma_{n}+\nabla \cdot\left(\sigma_{n} w_{n-1}\right)=G_{n-1} \text { en } \Omega \quad \text { y } \quad \int_{\Omega} \sigma_{n} d x=0
$$

donde $G_{n-1}=\pi_{n-1}-\bar{\rho} K$ y $w_{n-1}=\frac{\zeta+\mu / 3}{\bar{\rho} \mu} u_{n-1}$.

Para la inicialización del método iterativo tomaremos:

$$
\sigma_{0}=\theta_{0}=0, v_{0}=0 \quad \text { y } \quad \pi_{0}=\bar{\rho} K
$$

La idea es probar que una solución de nuestro problema se obtiene como límite de soluciones de problemas aproximados $A_{n}$ y $B_{n}$. La existencia y unicidad de solución de dichos problemas se tendrá por los Lemas 2.2 y 2.3 .

En la demostración seguiremos los siguientes pasos:

1) Acotación de $\theta_{n-1}, F_{n-1}$ en función de $\left(u_{n-1}, \pi_{n-1}\right)$ y $\sigma_{n-1}$ :

Suponga que $\left(u_{n-1}, \pi_{n-1}\right)$ y $\sigma_{n-1}$ satisfacen las estimaciones:

$$
\left\{\begin{aligned}
\left|u_{n-1}\right|_{2,4}+\left|\pi_{n-1}\right|_{1,4} & \leq \frac{3}{2} C_{2} \bar{\rho}|f|_{4}, \\
\left|\nabla \sigma_{n-1}\right|_{4} & \leq \frac{2 C_{2} \bar{\rho}}{K}|f|_{4} .
\end{aligned}\right.
$$

donde $C_{2}>0$, es la constante generada por el lema de Cattabriga-Solonnikov, dependiendo únicamente de $\Omega$. Entonces de la definición de $\theta_{n-1}$ y $F_{n-1}$ se deduce que

$$
\left|\theta_{n-1}\right|_{1,4} \leq C_{1}\left|\nabla \theta_{n-1}\right|_{4} \leq C_{1} C_{2} \bar{\rho}|f|_{4} \frac{7}{2(\zeta+\mu / 3)}
$$

$\mathrm{y}$

$$
\left|F_{n-1}\right|_{4} \leq\left(\bar{\rho}+C_{1} C_{4}\left|\nabla \sigma_{n-1}\right|_{4}\right)\left(\frac{C_{4}}{\mu^{2}}\left|u_{n-1}\right|_{2,4}^{2}+|f|_{4}\right) .
$$

Lo cual conduce a:

$$
\left|F_{n-1}\right|_{4} \leq\left(1+\frac{2 C_{1} C_{2} C_{4}}{K}|f|_{4}\right)\left(1+\frac{9}{4} C_{4}\left(\frac{C_{2} \bar{\rho}}{\mu}\right)^{2}|f|_{4}\right) \bar{\rho}|f|_{4} .
$$

\section{Existencia de $A_{n}$ y $B_{n}$ :}

De (21), (23) y (18) se tiene respectivamente que $\theta_{n-1} \in W^{1,4}(\Omega), F_{n-1} \in\left(L^{4}(\Omega)\right)^{3}$ y $\int_{\Omega} \theta_{n-1} d x=0, \forall n \geq 2$.

Si $\partial \Omega$ es de clase $\mathcal{C}^{2}$, entonces por el Lema 2.2 existe una única solución del Problema $A_{n}$, $\left(u_{n}, \pi_{n}\right) \in\left(W^{2,4}(\Omega)\right)^{3} \times W^{1,4}(\Omega)$.

Por otro lado, el Lema 2.3 y las condiciones $A_{i}<1$ aseguran la existencia de una solución $\sigma_{n}$ del Problema $B_{n}$, tomando

$$
a=K, b=\left(\frac{\zeta+\mu / 3}{\bar{\rho} \mu}\right) u_{n-1}, \psi=\sigma_{n} \quad \text { y } \quad G=\pi_{n-1}-\bar{\rho} K
$$


donde se verifica la condición (6). En efecto,

$$
\begin{aligned}
a-7 C_{1} C_{4} \mid & \left.\nabla(\nabla \cdot b)\right|_{4}-4 C_{4}|b|_{2,4} \\
& =k-7 C_{1} C_{4}\left(\frac{\zeta+\mu / 3}{\overline{\rho \mu}}\right)\left|\nabla\left(\nabla \cdot u_{n-1}\right)\right|_{4}-4 C_{4}\left(\frac{\zeta+\mu / 3}{\bar{\rho} \mu}\right)\left|u_{n-1}\right|_{4} \\
& =K-C_{4}\left(\frac{\zeta+\mu / 3}{\bar{\rho} \mu}\right)\left\{7 C_{1}\left|\nabla\left(\nabla \cdot u_{n-1}\right)\right|_{4}+4\left|u_{n-1}\right|_{2,4}\right\} \\
& \geq K-C_{4}\left(\frac{\zeta+\mu / 3}{\bar{\rho} \mu}\right)\left\{7 C_{1}\left|\nabla\left(\nabla \cdot u_{n-1}\right)\right|_{4}+6 C_{2} \bar{\rho}|f|_{4}\right\} \\
& =K-C_{4}\left(\frac{\zeta+\mu / 3}{\bar{\rho} \mu}\right) 7 C_{1} C_{2} \bar{\rho}|f|_{4} \frac{7}{2(\zeta+\mu / 3)}-6 C_{2} C_{4}\left(\frac{\zeta+\mu / 3}{\bar{\rho} \mu}\right) \bar{\rho}|f|_{4} \\
& =K-\frac{49}{2 \mu} C_{1} C_{2} C_{4}|f|_{4}-6 C_{2} C_{4} \frac{(\zeta+\mu / 3)}{\mu}|f|_{4} \\
& =K-C_{2} C_{4}|f|_{4}\left\{\frac{49 C_{1}}{2 \mu}+6\left(\frac{\zeta+\mu / 3}{\bar{\rho} \mu}\right)\right\} \\
& =K\left(1-\frac{A_{1}}{2}\right)>0 .
\end{aligned}
$$

Luego, $\sigma_{n}$ es la única solución, $\sigma_{n} \in D(L)\left(=\left\{\psi \in W^{1,4}(\Omega): L \psi \in W^{1,4}(\Omega), \int_{\Omega} \psi=0\right\}\right)$, tal que:

$$
L \sigma_{n}=K \sigma_{n}+\left(\frac{\zeta+\mu / 3}{\bar{\rho} \mu}\right) \nabla \cdot\left(\sigma_{n} u_{n-1}\right)=\pi_{n-1}-K \bar{\rho}
$$

\section{Verificación de la acotación de $(20)$ para $\left(u_{n}, \pi_{n}\right)$ y $\sigma_{n}$ :}

Se pretende demostrar (20) por inducción, es decir, supuesto cierto (20) queremos obtener (20) cambiando $n-1$ por $n$.

De la desigualdad:

$$
|\nabla G|_{4}^{4} \geq a^{3}\left[a-7 C_{1} C_{4}|\nabla(\nabla \cdot b)|_{4}-4 C_{4}|b|_{2,4}\right]|\nabla \psi|_{4}^{4}
$$

que aparece al final de la demostración del Lema 2.3, considere $a, G, b$ y $\psi$ como antes, así se obtiene

$$
K^{3}\left[K-7 C_{1} C_{4}\left(\frac{\zeta+\mu / 3}{\bar{\rho} \mu}\right)\left|\nabla\left(\nabla \cdot u_{n-1}\right)\right|_{4}-4 C_{4}\left(\frac{\zeta+\mu / 3}{\bar{\rho} \mu}\right)\left|u_{n-1}\right|_{2,4}\right]\left|\nabla \sigma_{n}\right|_{4}^{4} \geq\left|\nabla \pi_{n-1}\right|_{4}^{4} .
$$

Por otro lado, sustituyendo en la estimativa del Lema 2.2 las desigualdades (20), (21) y (22), y teniendo en cuenta la hipótesis $A_{2}<1$, se tiene que 


$$
\begin{aligned}
\left|u_{n}\right|_{2,4}+\left|\pi_{n}\right|_{1,4} & \leq C_{2}\left(\left|F_{n-1}\right|_{4}+\left|\sigma_{n-1}\right|_{1,4}\right) \\
& \leq\left[\frac{7 C_{1} C_{2}}{2(\zeta+\mu / 3)}+\left(1+\frac{2}{K} C_{1} C_{2} C_{4}|f|_{4}\right)\left(1+\frac{9}{4} C_{4}\left(C_{2} \frac{\bar{\rho}}{\mu}\right)^{2}|f|_{4}\right)\right] \bar{\rho} C_{2}|f|_{4} \\
& \leq\left(\frac{A_{2}}{2}+1\right) C_{2} \bar{\rho}|f|_{4} \\
& \leq \frac{3}{2} C_{2} \bar{\rho}|f|_{4}
\end{aligned}
$$

Finalmente, usando las desigualdades (20) y (24), teniendo en cuenta la hipótesis $A_{1}<1$, se concluye que

$$
\left|\nabla \sigma_{n}\right|_{4} \leq 2 C_{2} \frac{\bar{\rho}}{K}|f|_{4}
$$

En consecuencia, las soluciones $\left(u_{n}, \pi_{n}\right), \sigma_{n}$ están acotadas en $\left(W^{2,4}(\Omega)\right)^{3} \times W^{1,4}(\Omega) \times$ $W^{1,4}(\Omega)$, como en $(20)$.

\section{Convergencia de $\left(u_{n}, \pi_{n}\right)$ y $\sigma_{n}$ :}

Denotamos

$$
\begin{aligned}
& u_{n+1}^{\prime}=u_{n+1}-u_{n} \\
& \pi_{n+1}^{\prime}=\pi_{n+1}-\pi_{n} \\
& \sigma_{n+1}^{\prime}=\sigma_{n+1}-\sigma_{n}
\end{aligned}
$$

Restando las ecuaciones que verifican $\left(u_{n+1}, \pi_{n+1}\right)$ y $\sigma_{n+1}$ de las que verifican $\left(u_{n}, \pi_{n}\right)$ y $\sigma_{n}$, de los lemas 2.2 y 2.3 , (11) dado que se verifica la condición $A_{1}<1$, se deduce que:

$$
\left|u_{n+1}^{\prime}\right|_{1,2}+\left|\pi_{n+1}^{\prime}\right|_{2} \leq C_{2}\left\{\left|F_{n}-F_{n-1}\right|_{-1,2}+\left|\theta_{n}-\theta_{n-1}\right|_{2}\right\}
$$

y

$$
\left|\sigma_{n+1}^{\prime}\right|_{2} \leq \frac{4}{K} C_{2}\left(\frac{\zeta+\mu / 3}{\mu}\right)\left(C_{1} C_{4}+C_{5}\right)|f|_{4}\left|u_{n}^{\prime}\right|_{1,2}+\frac{2}{K}\left|\pi_{n}^{\prime}\right|_{2}
$$

donde $C_{5}$ es la constante de inmersión de $H^{1}(\Omega)$ en $L^{4}(\Omega)$.

Por otro lado, de la definición de $F_{n}$ se obtiene que:

$$
F_{n}-F_{n-1}=\frac{1}{\mu^{2}}\left(\bar{\rho}+\sigma_{n-1}\right)\left(u_{n}^{\prime} \cdot \nabla u_{n}+u_{n-1} \cdot \nabla u_{n}^{\prime}\right)+\sigma_{n}^{\prime}\left(\frac{1}{\mu^{2}} u_{n} \cdot \nabla u_{n}-f\right)
$$

lo que junto con la definición de $\theta_{n}$ y $G_{n}$ nos conduce a

$$
\left\{\begin{aligned}
\left|F_{n}-F_{n-1}\right|_{-1,2} \leq & \frac{1}{\mu^{2}}\left(2 \bar{\rho} C_{4}\left|u_{n}\right|_{2,4}+\left|u_{n}\right|_{1,4}\left|\sigma_{n-1}\right|_{1,4}+C_{4}\left|\sigma_{n-1}\right|_{1,4}\left|u_{n}\right|_{1,4}\right)\left|u_{n}^{\prime}\right|_{1,2} \\
& +\left(\frac{1}{\mu^{2}} C_{4}^{2}\left|u_{n}\right|_{2,4}^{2}+|f|_{4} K_{1}\right)\left|\sigma_{n}^{\prime}\right|_{2} \\
\left|\theta_{n}-\theta_{n-1}\right|_{2} \leq & \frac{\mu}{\zeta+\mu / 3}\left\{K\left|\sigma_{n}^{\prime}\right|_{2}+\left|\pi_{n}^{\prime}\right|_{2}\right\} \\
\left|G_{n}-G_{n-1}\right|_{2} & =\left|\pi_{n}^{\prime}\right|_{2}
\end{aligned}\right.
$$


Uniendo finalmente las relaciones anteriores y (20) se concluye

$$
\begin{aligned}
& \left|u_{n+1}^{\prime}\right|_{1,2}+\left|\nabla \pi_{n+1}^{\prime}\right|_{-1,2}+\left|\sigma_{n+1}^{\prime}\right|_{2} \leq \\
& C_{2}|f|_{4}\left\{\frac{4}{K}\left(\frac{\zeta+\mu / 3}{\mu}\right)\left(C_{1} C_{4}+C_{5}\right)+3 C_{2}\left(\frac{\bar{\rho}}{\mu}\right)^{2}\left(C_{4}+\left(1+C_{4}\right) \frac{C_{1} C_{2}}{K}|f|_{4}\right)\right\}\left|u_{n}^{\prime}\right|_{1,2} \\
& \quad+\left\{\frac{2}{K}+C_{2} \frac{\mu}{\zeta+\mu / 3}\right\}\left|\pi_{n}^{\prime}\right|_{2}+\left\{|f|_{4}\left(K_{1}+\frac{9}{4}\left(C_{2} C_{4} \frac{\bar{\rho}}{\mu}\right)^{2}|f|_{4}\right)+\frac{\mu K}{\zeta+\mu / 3}\right\} C_{2}\left|\sigma_{n}^{\prime}\right|_{2}
\end{aligned}
$$

y con las condiciones de los $A_{i}$, para $i=3,4,5$, queda claro que

$$
\left|u_{n+1}^{\prime}\right|_{1,2}+\left|\nabla \pi_{n+1}^{\prime}\right|_{-1,2}+\left|\sigma_{n+1}^{\prime}\right|_{2} \leq A_{3}\left|u_{n}^{\prime}\right|_{1,2}+A_{4}\left|\nabla \pi_{n}^{\prime}\right|_{-1,2}+A_{5}\left|\sigma_{n}^{\prime}\right|_{2}
$$

Para obtener esta numeración, se ha usado el siguiente resultado el cual puede encontrarse en [12]. Note que fue necesario conseguir estimativas para $\pi_{n}^{1}$ en $w^{-1,2}$ siempre que $\pi_{n}^{1}$ posea norma en $L^{2}(\Omega)$.

Lema 3.4 (Necas) Existe una constante $C_{6}>0$ tal que:

$$
|q|_{0} \leq C_{6}|\nabla q|_{-1,2} \quad \forall q \in L_{0}^{2}(\Omega) .
$$

Entonces, como se ha supuesto que $A_{i}<1$, para $i=3,4,5$, se puede deducir la convergencia fuerte en las respectivas normas de $H_{0}^{1}(\Omega), L^{2}(\Omega), L^{2}(\Omega)$, mediante un procedimiento estándar, a partir del método iterativo comparativo para toda la sucesión $\left(u_{n}, \pi_{n}, \sigma_{n}\right)$ a un límite que llamaremos $(u, \pi, \sigma)$.

Tomando entonces límite cuando $n \rightarrow \infty$ en las condiciones del Problema $A_{n}$, (19) y las definiciones de $F_{n-1}, \theta_{n-1}$ y $G_{n-1}$, se encuentra que $(u, \pi)$ y $\theta$ satisfacen:

$$
\left\{\begin{array}{l}
-\Delta u+\nabla \pi=-\frac{\bar{\rho}+\sigma}{\mu^{2}} u \cdot \nabla u+(\sigma+\bar{\rho}) f \\
\nabla \cdot u=\frac{\mu}{(\zeta+\mu / 3)} K(\sigma+\bar{\rho})-\pi \\
K \sigma+\frac{\zeta+\mu / 3}{\bar{\rho} \mu} \nabla \cdot(\sigma u)=\pi-\bar{\rho} K, \text { con } \int_{\Omega} \sigma d x=0
\end{array}\right.
$$

Como dicho problema es equivalente a (14), se observa que $(v, p)$ (para $v=u / \mu$ y $\rho=$ $\bar{\rho}+\sigma)$ es una solución del problema (14)-(2). Además, las estimaciones (15) y (16) se obtienen fácilmente de (20). Solo resta demostrar la estimativa puntual inferior (17). Dicha estimativa se deduce de que $A_{2}<1$, de la siguiente forma:

$$
\begin{aligned}
\rho & \equiv \bar{\rho}+\sigma \geq \bar{\rho}-|\sigma|_{\infty} \geq \bar{\rho}-C_{4}|\sigma|_{1,4} \\
& \geq \bar{\rho}-C_{1} C_{4}|\nabla \sigma|_{4} \geq \bar{\rho}-\frac{2 C_{1} C_{2} C_{4}}{K}|f|_{4} \bar{\rho}=\bar{\rho}\left(1-\frac{2 C_{1} C_{2} C_{4}}{K}|f|_{4}\right) \\
& >\bar{\rho}
\end{aligned}
$$

donde hemos usado la desigualdad de Poincaré desde que $\int_{\Omega} \sigma d x=0$.

Lo que demuestra el resultado principal. 


\section{Conclusiones}

\section{Conclusión 1.}

Respecto al estudio de la ecuación (1), y del Teorema 3.2, podemos concluir que no solo existen soluciones estacionarias del problema, si no, que estas son únicas para cada fuerza externa $f \in\left(L^{4}\right)^{3}$ y densidad $\rho \in W^{1,4}(\Omega)$ fija. Esto es debido a que si suponemos que existen dos soluciones $\left(v_{i}, \rho\right) \in\left(W^{2,4}(\Omega)\right)^{3} \times W^{1,4}(\Omega)$ para $i=1,2$ con la misma fuerza externa $f \in\left(L^{4}(\Omega)\right)^{3}$ y la misma densidad $\rho$, entonces, al comparar las velocidades, obtenemos el sistema

$$
\left\{\begin{array}{r}
-\nabla \cdot\left(\mu\left(\nabla w+\nabla w^{t}\right)+\lambda(\nabla \cdot w) I d\right)+\nabla p+\rho w \cdot \nabla w=0 \text { en } \Omega, \\
\nabla \cdot(\rho w)=0 \text { en } \Omega,
\end{array}\right.
$$

con $w=v_{1}-v_{2}$, donde, por el Teorema 3.2 sobre el sistema (27) con fuerza externa nula, se tiene que

$$
|w|_{2,4} \leq 0
$$

es decir, $v_{1}=v_{2}$ en $\left(W^{2,4}(\Omega)\right)^{3}$.

\section{Conclusión 2.}

El caso barotrópico general, i.e., donde $p=p(\rho)$ admite un análisis análogo vía el mismo método, realizando previamente la siguiente modificación:

$$
\nabla p(\rho)=p^{\prime}(\rho) \nabla \rho=p^{\prime}(\bar{\rho}) \nabla \rho+\left(p^{\prime}(\bar{\rho})\right) \nabla \rho
$$

donde $p^{\prime}(\bar{\rho}) \equiv$ constante, al ser el valor de la función $p$ en el punto $\bar{\rho}=\frac{1}{|\Omega|} \int_{\Omega} \rho d x$.

Sustituyendo $\nabla p(\rho)$ en la ecuación (14) por $p^{\prime}(\bar{\rho}) \nabla \rho$, pasando $p^{\prime}(\rho)-p^{\prime}(\bar{\rho}) \nabla \rho$ al segundo miembro, es decir:

$$
-\mu \Delta u-(\zeta+\mu / 3) \nabla(\nabla \cdot v)+p^{\prime}(\bar{\rho}) \nabla \rho=\left[p^{\prime}(\bar{\rho})-p^{\prime}(\rho)\right] \nabla \rho-\rho[(v \cdot \nabla) v-f]
$$

y la $F$ del problema A quedaría de la forma:

$$
F=-\frac{\sigma+\bar{\rho}}{\mu^{2}} u \cdot \nabla u+(\sigma+\bar{\rho}) f+\left[p^{\prime}(\bar{\rho})-p^{\prime}(\sigma+\bar{\rho})\right] \nabla \sigma
$$

Si $p \in \mathcal{C}^{2}(\Omega)$, se puede escribir:

$$
p^{\prime}(\bar{\rho})-p^{\prime}(\bar{\rho}+\sigma)=-p^{\prime \prime}(\xi) \theta
$$

donde $\xi$ es un valor intermedio entre $\bar{\rho}$ y $\bar{\rho}+\sigma$ (variable), con lo que:

$$
F=\frac{\sigma+\bar{\rho}}{\mu^{2}} v \cdot \nabla v-(\sigma+\bar{\rho}) f-p^{\prime \prime}(\xi) \sigma \nabla \sigma
$$

Se introducirán entonces las modificaciones correspondientes en la escritura de los $A_{i}$, para que a partir de las nuevas acotaciones de $F_{n-1}$, se pueda garantizar las mismas desigualdades necesarias para la convergencia del método de las aproximaciones sucesivas.

\section{Conclusión 3.}

Se obtiene unicidad de solución para la linealización de las ecuaciones de Navier-stokes barotrópicas cerca de un flujo en un ambiente ideal dado. La novedad se presenta que la formulación de la solución en velocidad-presión y no en velocidad-densidad, como es usualmente estudiado. 


\section{Referencias bibliográficas}

[1] Adams, R. y Fournier, J. (2003). Sobolev Spaces. (Second ed.). New York, United States: Academic Press.

[2] Aris, R. (1990). Vectors, Tensors and the Basic Equations of Fluid Mechanics. New York, United States: Dover.

[3] Batchelor, G. (1994). An Introduction to Fluid Dynamics. Cambridge, United Kingdom: Cambridge University Press.

[4] Coimbra, A. (1967). Mecânica dos Meios Cointínuos. Rio de Janeiro, Brasil: Ao Livro Técnico.

[5] Dufour, G. (2005). Modélisation multi-fluide eulérienne pour les écoulements diphasiques à inclusions dispersées ( $\mathrm{PhD}$ thesis). Université Paul-Sabatier Toulouse-III, Toulouse, France.

[6] Fox, R., McDonald, A. y Pritchard, P. (2006). Introdução à Mecânica dos Fluidos. Rio de Janeiro, Brasil: LTC.

[7] Gilbarg, D. y Trudinger, N. (1983). Elliptic Partial Differential Equations of Second Order. Berlin, Alemania: Springer.

[8] Landau, L. y Lifshitz. E. M. (1959). Fluid Mechanics. New York, United States: Pergamon.

[9] Liepman, W. H., Roshko. A. (1957). Elements of Gas Dynamics. New York, United States: Wiley.

[10] Lions, P. (1996). Mathematical Topics in Fluids Mechanics, Vol. 1, Incompressible Models. Oxford, United Kingdom: Oxford University Press.

[11] Lukaszewicz, G. y Kalita, P. (2016). Navier-Stokes equations. An introduction with applications. Advances in Mechanics and Mathematics.(Vol. 34). New York, United States: Springer.

[12] Necas, J. (1962). Sur une méthode pour résoudre les edp du style elliptique voisine de la variationnelle. Annali della Scuola Normale Superiore di Pisa, 16(4), p. 305-326

[13] Padula, M. (1982). Existence and Uniqueness for Viscous Steady Compressible Motions. Archive for Rational Mechanics and Analysis, 97, 89-102.

[14] Renardy, M. y Rogers, R. (2004). An introduction to partial differential equations. (Second ed). New York, United States: Springer-Verlag.

[15] Stokes, G. (1880). Mathematical Physical Papers (vol. 1). Cambridge, United Kingdom: Cambridge University Press.

[16] Teman, R. (1979). Navier-Stokes equations: theory and numerical analysis. Vol. 2 of Studies in mathematics and its applications. California, United States: North-Holland Pub. Co.

[17] Valli, A. (1987). On the Existence of Stationary Solutions to Compressible Navier-Stokes Equations. Annales de l'Institut Henri Poincare (C) Non Linear Analysis, 4(1), 99-113.

[18] White, F. (2002). Mecânica dos Fluidos. Lisboa, Portugal: McGraw-Hill.

[19] Williams, F. (1985). Combustion Theory (second edition). California, United States: The Benjamin Cummings. 